\title{
BMJ Open Protocol for a multicentre, parallel- group, open-label randomised controlled trial comparing ferric carboxymaltose with the standard of care in anaemic Malawian pregnant women: the REVAMP trial
}

Martin N Mwangi (D) , ,2 Glory Mzembe, ${ }^{1,2}$ Ernest Moya, ${ }^{1,2}$ Sabine Braat (D) ,3,4 Rebecca Harding, ${ }^{5}$ Bjarne Robberstad, ${ }^{6}$ Julie Simpson, ${ }^{3}$ William Stones (1) , ${ }^{7}$ Stephen Rogerson, ${ }^{4,8}$ Kabeya Biselele, ${ }^{9}$ Jobiba Chinkhumba (D) ,10 Leila Larson, ${ }^{11}$ Ricardo Ataíde, ${ }^{4,5}$ Kamija S Phiri, ${ }^{1,2}$ Sant-Rayn Pasricha ${ }^{5,12}$

To cite: Mwangi MN, Mzembe G, Moya E, et al. Protocol for a multicentre, parallel-group, open-label randomised controlled trial comparing ferric carboxymaltose with the standard of care in anaemic Malawian pregnant women: the REVAMP trial. BMJ Open 2021;11:e053288. doi:10.1136/ bmjopen-2021-053288

- Prepublication history and additional supplemental material for this paper are available online. To view these files, please visit the journal online (http://dx.doi.org/10.1136/ bmjopen-2021-053288)

KSP and S-RP are joint senior authors.

Received 10 May 2021 Accepted 25 October 2021

Check for updates

(C) Author(s) (or their employer(s)) 2021. Re-use permitted under CC BY. Published by BMJ.

For numbered affiliations see end of article.

Correspondence to Dr Martin N Mwangi; mmwangi@true.mw

\section{ABSTRACT}

Introduction Anaemia in pregnancy remains a critical global health problem, affecting $46 \%$ of pregnant women in Africa and $49 \%$ in Asia. Oral iron therapy requires extended adherence to achieve correction of anaemia and replenishment of iron stores. Ferric carboxymaltose (FCM) is a recently established intravenous iron formulation associated with substantial advantages in safety, speed of delivery and total dose deliverable in a single infusion. We aim to determine whether FCM given once during the second trimester of pregnancy compared with standard oral iron distributed through routine antenatal services is effective and safe for treatment of moderate to severe maternal anaemia in sub-Saharan Africa.

Methods and analysis The randomized controlled trial of the effect of intravenous iron on anaemia in Malawian pregnant women (REVAMP) is a two-arm confirmatory individually randomised trial set in Blantyre and Zomba districts in Malawi. The trial will randomise 862 women in the second trimester of pregnancy with a capillary haemoglobin concentration below $100.0 \mathrm{~g} / \mathrm{L}$. The study comprises two arms: (a) intravenous FCM $(20 \mathrm{mg} / \mathrm{kg}$ up to $1000 \mathrm{mg}$ ) given once at randomisation, and (b) standard of care oral iron $(65 \mathrm{mg}$ elemental iron two times per day) for 90 days (or the duration of pregnancy, whichever is shorter) provided according to local healthcare practices. Both arms receive sulfadoxine-pyrimethamine as intermittent preventive treatment in pregnancy. The primary outcome is the prevalence of anaemia $(\mathrm{Hb}<110.0 \mathrm{~g} / \mathrm{L})$ at 36 weeks' gestation. Secondary outcomes include birth weight, gestation duration and safety outcomes, including clinical malaria, serious perinatal events and postpartum haematologic and healthrelated outcomes in the mother and child.

Ethics and dissemination Ethical approval was granted by the Research Ethics Committee (COMREC P.02/18/2357) in Malawi and the Human Research Ethics Committee (WEHI: 18/02), Melbourne, Australia. The protocol is registered with the Australian and New Zealand

\section{Strengths and limitations of this study}

- Eligibility for inclusion-moderate or severe anaemia-is assessed by capillary haemoglobin estimation, a method that could be deployed at the local health centre level.

- The trial follows pregnant women and their babies through delivery and into the postpartum period enabling the assessment of antenatal and postnatal effects of the intervention.

- This trial uses a modern intravenous iron formulation for the treatment of anaemia in pregnancy which enables a high dose iron infusion (up to $1000 \mathrm{mg}$ ) to be infused in a single dose.

- The trial will measure a broad range of haematologic, safety and clinical-efficacy outcomes.

- This trial is open-label, and participants will know the trial intervention to which they have been randomised.

Clinical Trials Registry. The results will be shared with the local community that enabled the research, and also to the international fora.

Trial registration number ACTRN12618001268235; Pre-results.

\section{INTRODUCTION}

Anaemia during pregnancy remains a critical global health problem. Almost $40 \%$ of pregnant women worldwide are anaemic, including $46 \%$ of pregnant women in Africa and $49 \%$ in Asia. ${ }^{1}$ Anaemia in pregnancy is very common and mostly results from iron deficiency and is associated with critical risks for both mother (eg, life-threatening complications of postpartum haemorrhage) 
and child (especially prematurity and low birth weight, ${ }^{2}$ which are associated with increased risk of neonatal and infant mortality, ${ }^{3}$ and reduced iron stores in infancy with increased risk of subsequent anaemia and impaired development). ${ }^{4}$ Severe anaemia in pregnancy is associated with a significantly increased risk of maternal mortality. ${ }^{5}$ Worldwide, $15 \%-20 \%$ of births (>20 million annually, including 13\% in sub-Saharan Africa) are low birth weight. At the same time, each year, over 1 million children die from preterm birth complications, making prevention and treatment of antenatal anaemia a crucial component of efforts to reduce low birth weight.

Systematic reviews confirm likely benefits from iron on maternal outcomes, including anaemia ( $70 \%$ reduction) and trends towards favourable infant outcomes, including increased birth weight and extended gestation duration. ${ }^{6}$ A trial of oral iron compared with placebo in Kenyan pregnant women confirmed a benefit from iron on birth weight and gestation duration. ${ }^{7}$ Global recommendations for managing anaemia in pregnancy in lower and middle income countries currently advise that women be treated with high-dose daily oral iron (120 mg of elemental iron) supplementation for 3 months. ${ }^{89}$ However, oral iron is often poorly tolerated due to gastrointestinal adverse effects, ${ }^{10}$ limiting adherence; lower doses with intermittent dosing have been used in non-pregnant women. ${ }^{11}$ Delivery of iron during pregnancy requires high-quality performance of the primary health system. In Malawi, $10.6 \%$ of women were reported to take no iron during pregnancy, and $37.1 \%$ take less than 60 doses, with only $33.4 \%$ taking the recommended 90 or more doses. ${ }^{12}$ Even when delivered, oral iron frequently fails to correct anaemia. For example, $61 \%$ of Gambian pregnant women who received iron at week 20 of gestation-when their $\mathrm{Hb}$ was lower than $100.0 \mathrm{~g} / \mathrm{L}$-still had a $\mathrm{Hb}<100.0 \mathrm{~g} / \mathrm{L}$ at 30 weeks' gestation. ${ }^{13}$ Finally, women may only present for their first antenatal visit late in the second trimester, curtailing the time available to optimise iron stores with oral iron to improve foetal development and risks associated with delivery; for example, in Malawi, fewer than $30 \%$ of pregnant women attend before the 16 th week of gestation. $^{14}$

Over the past decade, there have been dramatic improvements in parenteral (intravenous) iron therapeutics. Ferric carboxymaltose (FCM) is a widely used formulation that can be administered in a short period (15 min) and at large doses (up to $1000 \mathrm{mg}$ ) in a single infusion. ${ }^{15}{ }^{16} \mathrm{FCM}$ has revolutionised the treatment of iron-deficiency anaemia in high-income country settings and is widely used in outpatient settings, emergency departments and primary care. ${ }^{17}$ In pregnancy, intravenous iron has been reported to be superior to oral iron in improving haemoglobin concentrations and may even increase birth weight. ${ }^{18}$ Intravenous iron is increasingly recommended as a suitable first-line option for women with moderate or severe iron-deficiency anaemia beyond the first trimester of pregnancy. ${ }^{19}$ Studies have evaluated the role of older forms of intravenous iron in pregnancy in low-income settings such as India, ${ }^{20} 21$ while other studies have demonstrated the feasibility of using FCM in the postpartum period in sub-Saharan Africa ${ }^{22}$ but modern formulations capable of delivering a rapid total-dose infusion have not yet been studied in women in pregnancy in low-income countries.

It remains challenging to directly measure iron status in the field, as biomarkers generally require analysis using centralised laboratory infrastructure. In contrast, point-of-care measurement of capillary haemoglobin is a feasible field-friendly testing strategy that can be deployed with existing technology, of which HemoCue point-of-care devices are a good example. Screen-andtreat approaches for anaemia in pregnancy must rely on haemoglobin measurement until point-of-care assays for iron status become more widely available.

FCM presents a practical opportunity for rapid correction of moderate and severe antenatal anaemia in a single dose, potentially improving maternal and neonatal clinical outcomes for pregnant women. It is, therefore, urgent to test this therapy in a low-income field setting where the burden of anaemia-related disease is heaviest. In Malawian pregnant women with moderate or severe anaemia during the second trimester of pregnancy, we aim to evaluate whether a single dose of intravenous FCM is superior to oral iron provided via standard-of-care approaches.

\section{METHODS AND ANALYSIS \\ Patient and public involvement}

We held discussions with national policy stakeholders during the study planning stage, including the Ministry of Health. We discussed how the research might align with national research and health service priorities. Local community engagement was done via public meetings; the potential participants were first involved in the study's design during these meetings. We discussed the study with the traditional leaders, including traditional authorities, group village headmen $(\mathrm{GVH})$, the village development committee and ward councillors (political figures). The GVH were requested to cascade the information to the village chiefs who took the information to the community. Health workers were accessed via the District Health Offices in Blantyre and Zomba districts. Health workers from the participating health facilities, including the health surveillance assistants who directly work with the community, were informed about the proposed research and discussions on priorities beneficial to the community were discussed. The public, potential participants and health workers identified malaria and anaemia as major public health issues in the community. Women outlined their experiences with iron supplements during their past pregnancies and identified the development of tolerable iron formulations as a research priority. Dissemination of findings at the national and community levels will follow 
the schema used during study design and inception as outlined above.

\section{Trial objectives}

The primary objective of the trial is to determine whether, in Malawian women in the second trimester of pregnancy with moderate or severe anaemia, a single dose of FCM up to $1000 \mathrm{mg}$ is superior to standardof-care (ie, oral iron provided through local health services), in reducing maternal anaemia before delivery (at 36 weeks' gestation).

The secondary objectives are:

During pregnancy and up to 1 month postpartum, to determine the effects of FCM (compared with standardof-care) on:

\section{Effectiveness}

1. Maternal haemoglobin concentration and iron status (measured through iron biomarkers).

2. Critical neonatal outcomes including birth weight (low birth weight), gestation duration (prematurity), small for gestational age and other perinatal outcomes.

Safety

1. Maternal and neonatal adverse events (AEs), including infection episodes, serious maternal complications and hypophosphatemia.

Exploratory objectives up to 1 month postpartum for subsequent hypothesis generation are related to maternal cognition, depression and fatigue, as well as costs of healthcare.

\section{Study design}

The Randomized controlled trial of the Effect of intraVenous iron on Anaemia in Malawian Pregnant women (REVAMP) is a multicentre, open-label, two-arm, parallel-group individually randomised controlled trial (RCT). An open-label (rather than blinded) design was selected as it was considered unfeasible to deliver the placebo intravenous infusions to Malawian pregnant women in this field-trial context (the drug is darkcoloured). The trial recruited pregnant women in their second trimester and is following them until 1 month postpartum, after which we will report on the primary and secondary objectives. Extended follow-up of mothers and infants to 12 months postpartum is planned, as is a range of exploratory economic, biological and clinical outcomes. These analyses are beyond the scope of this protocol, as are the exploratory outcomes collected up to 1 month postpartum, and they will be reported separately. The trial design is summarised in figure 1 .

\section{Study settings and participants}

In Malawi, the average gestation age at the first antenatal clinic (ANC) visit is 22 weeks, with $61 \%$ presenting before 24 weeks. The 2015-2016 DHS survey estimated a prevalence of anaemia $(\mathrm{Hb}<110.0 \mathrm{~g} / \mathrm{L})$ in pregnant women in Malawi at $45.1 \%$, including $22.4 \%$ of women with moderate or severe anaemia. ${ }^{12}$ The prevalence of low birth weight in Malawi was around $14 \%$ in 2015. ${ }^{23}$ Malawi is endemic for malaria and the prevalence of Plasmodium spp parasitaemia at the first ANC visit in the study site exceeds $15 \% .{ }^{24}$ About $87 \%$ of women consume at least one dose of intermittent preventive treatment during pregnancy (IPTp), but this falls to $30 \%$ for women taking three or more doses. ${ }^{12}$

The REVAMP study has two major research sites namely Limbe Health Centre and Zomba Central Hospital located in Blantyre and Zomba districts,

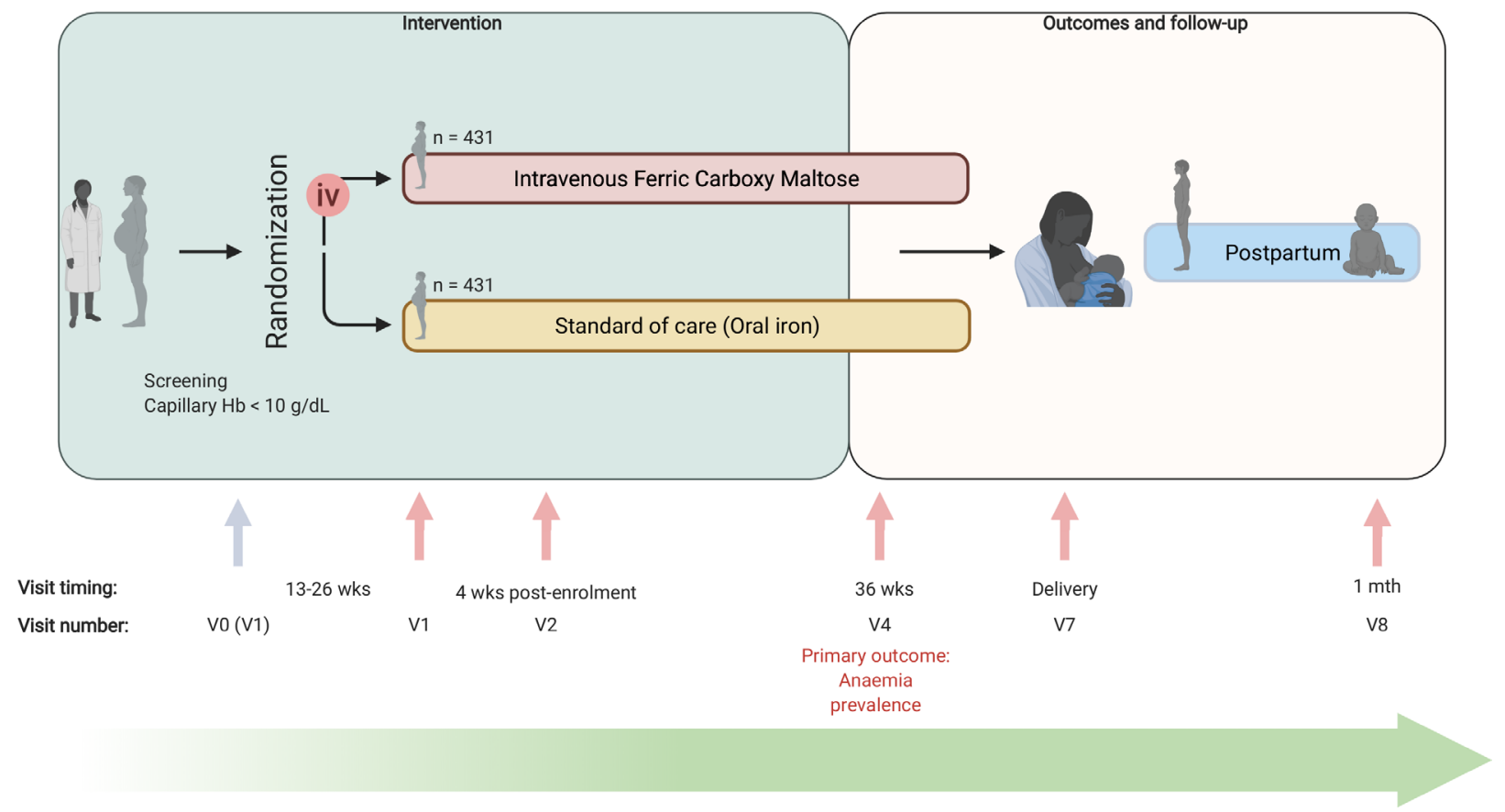

Figure 1 REVAMP trial schema. iv, intravenous; REVAMP, Randomized controlled trial of the Effect of intraVenous iron on Anaemia in Malawian Pregnant women; V0, visit 0; V1, visit 1; V2, visit 2; V4, visit 4; V7, visit 7; V8, visit 8; wks, weeks. 
respectively, in Southern Malawi. The two major sites act as the study coordination centres in the respective districts. The Blantyre site serves as the base for five urban health centres/ANCs, while the Zomba site serves as the base for nine (four urban and five rural) health centres/ANCs. Women are screened at the ANCs and referred to the coordinating research site for enrolment, treatment and follow-up visits. Deliveries occur in government-operated birth suites attached to the study site with referral to a district hospital where required for obstetric indications.

\section{Eligibility criteria}

Women are only randomised if they fulfil all inclusion criteria and none of the exclusion criteria.

\section{Inclusion criteria}

1. Confirmed singleton pregnancy at 13-26 weeks' gestation.

2. Moderate to severe anaemia (capillary haemoglobin $<100.0 \mathrm{~g} / \mathrm{L}$ measured by HemoCue $301+$ ) not clinically deemed to require an immediate blood transfusion. We excluded women with mild anaemia as thresholds to distinguish mild anaemia from health are indistinct, and because moderate and severe anaemia have an increased link to adverse maternal and child health outcomes. ${ }^{25}$

3. Negative malaria parasitaemia at screening and no positive malaria rapid diagnostic test within the previous 7 days.

4. Resident in the study catchment area of Blantyre and Zomba district.

5. Plan to deliver at a health facility.

6. Written informed consent, including assent if $<18$ years old (consent of a parent or guardian plus the participant's assent).

\section{Exclusion criteria}

1. Hypersensitivity to any of the study drugs.

2. Clinical symptoms of malaria or positive malaria rapid diagnostic test within the previous 7 days, or symptoms of bacterial infection, at screening.

3 . Any condition requiring hospitalisation or serious concomitant illness.

4. Chronic illness that may adversely affect foetal growth and viability.

5. Severe anaemia clinically judged as requiring a blood transfusion (usually $\mathrm{Hb}<50.0 \mathrm{~g} / \mathrm{L}$ ).

6. Pre-eclampsia at screening (new onset of hypertension, proteinuria and swelling in the legs, feet and hands).

\section{Trial interventions}

Participants are randomly assigned to receive one of the following interventions:

1. Intravenous iron: $\mathrm{FCM} 20 \mathrm{mg} / \mathrm{kg}$ up to $1000 \mathrm{mg}$, diluted in normal saline and given over $15 \mathrm{~min}$, once after randomisation. The study clinician administers the drug, and women remain under observation for $45 \mathrm{~min}$ after drug administration. Women receiving FCM are treated in rooms equipped with a trolley containing epinephrine, hydrocortisone, intravenous fluids and antihistamines, airway equipment and an oxygen concentrator. Staff are trained in the management of allergic reactions. FCM, manufactured by Vifor Pharma, was purchased at full-price from Aspen Pharma in Australia and shipped to Malawi.

2. Standard-of-care-oral iron treatment course: oral iron$200 \mathrm{mg}$ ferrous sulphate (approx. $65 \mathrm{mg}$ elemental iron) two times per day for 90 days or the remaining duration of pregnancy, whichever is shorter. Oral iron is provided by health workers using the same education messaging and similar packaging as provided by the routine health system to reflect real-life health service conditions.

Following national guidelines, both groups receive IPTp with $1500 \mathrm{mg}$ sulfadoxine and $75 \mathrm{mg}$ pyrimethamine (SP), as three tablets of SP of fixed dose $(500 \mathrm{mg} / 25 \mathrm{mg})$ at least three times during pregnancy, at least 4 weeks apart. The IPTp-SP course at 4weeks' post-randomisation is directly observed by study staff. HIV positive women in Malawi are not offered SP because they are already on cotrimoxazole prophylaxis. The baseline dose is also directly administered by study staff if the participant did not receive it through the health service. All participants also receive an insecticide-treated bed net.

\section{Randomisation, allocation concealment and blinding}

Participants are randomly allocated to one of the two treatments arms with 1:1 allocation via a computer-generated randomisation schedule of randomly permuted blocks stratified by research site (Blantyre, Zomba) to achieve a balance between the arms within each site. The randomisation list was generated by an independent statistician at the University of Melbourne (Australia).

Individual participant codes were pre-packed in sealed envelopes by an independent researcher not associated with the study and held securely at the research sites. Eligible participants who meet all inclusion/exclusion criteria are sequentially allocated participant identification numbers within the research site, and their allocation to study group is revealed after opening the corresponding sealed envelope. Although the trial is open-label, laboratory scientists measuring haemoglobin concentration, midwives collecting birth outcome data, and investigators and researchers in Australia (including data managers and statisticians in Melbourne) are blinded to the treatment allocation during the conduct of the trial until the database is locked and ready for unblinding.

\section{Recruitment and visits}

Table 1 shows the schedule of activities per visit for the mother, and table 2 shows the schedule of activities per visit for the neonate.

\section{Screening and enrolment of participants}

Study recruitment opened on 12 November 2018; the final participant was recruited on 2 March 2021. Study activities are detailed in tables 1 and 2. 


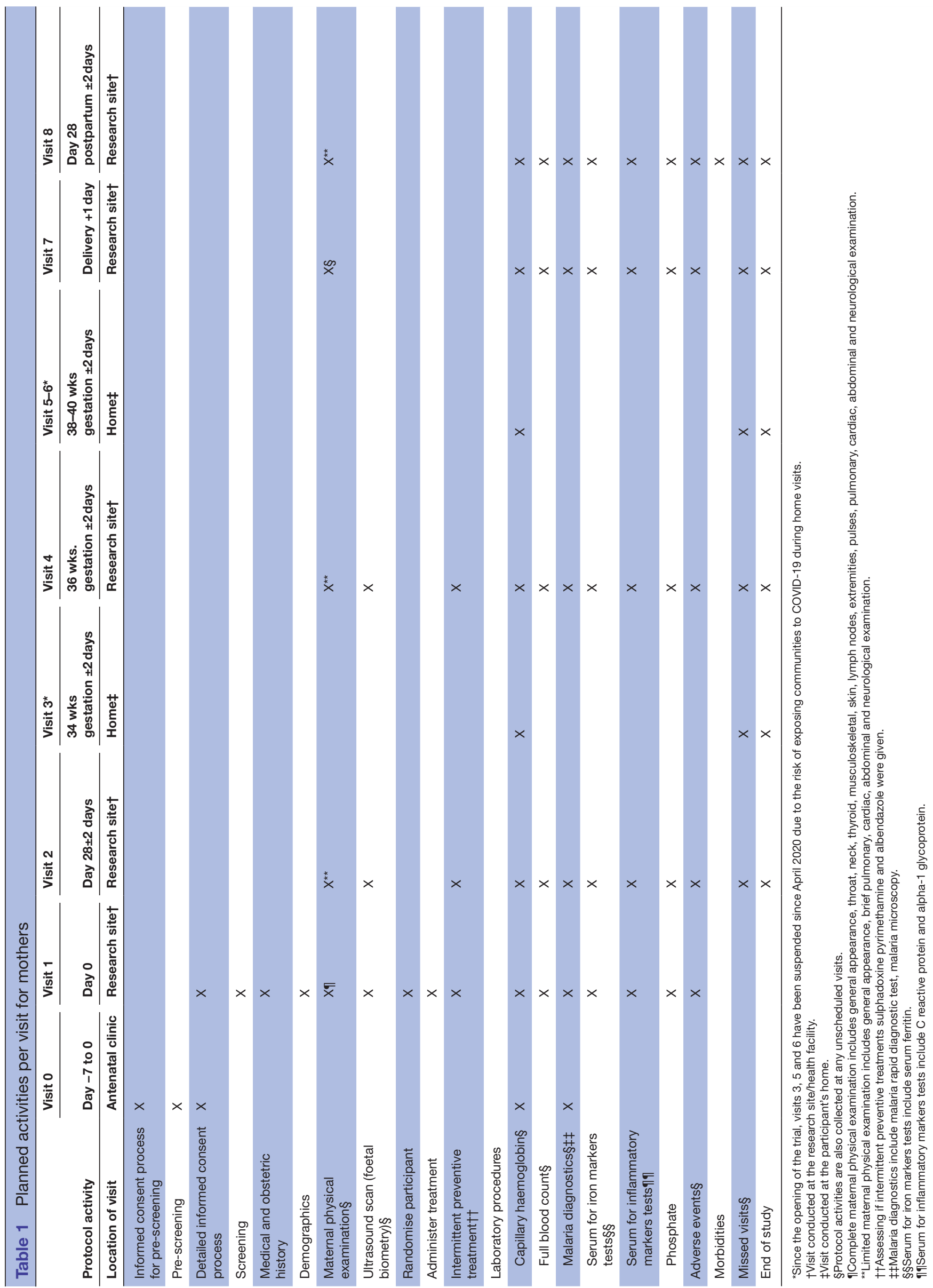


Table 2 Details of planned activities per visit for neonates

\begin{tabular}{|c|c|c|}
\hline \multirow[b]{2}{*}{ Protocol activity } & \multirow{2}{*}{$\begin{array}{l}\text { Visit } 7 \\
\text { Delivery }+1 \text { day }\end{array}$} & \multirow{2}{*}{$\begin{array}{l}\text { Visit } 8 \\
\text { Day } 28 \text { postpartum } \pm 2 \text { days }\end{array}$} \\
\hline & & \\
\hline Location of visit & Research site* & Research site* \\
\hline Physical examination and anthropometry†‡ & $X \S$ & $x ף$ \\
\hline \multicolumn{3}{|l|}{ Laboratory procedures } \\
\hline Full blood count $†$ & $\mathrm{X}^{\star *}$ & $\mathrm{X}$ \\
\hline Malaria diagnostics†† & $\mathrm{X}^{\star *}$ & $x$ \\
\hline Serum for iron markers tests $\ddagger \ddagger$ & $X^{\star *}$ & $\mathrm{X}$ \\
\hline Vaccination and Vit A supplementation status & & $x$ \\
\hline Adverse events $†$ & $x$ & $\mathrm{X}$ \\
\hline
\end{tabular}

*Visit done at the research site/ health facility.

†Protocol activities are also collected at any unscheduled visits.

$\ddagger$ Anthropometry includes assessing the infant's weight, length and head circumference.

$\S$ The complete physical examination includes general appearance, throat, neck, thyroid, musculoskeletal, skin, lymph nodes, extremities, pulses, pulmonary, cardiac, abdominal and neurological examination.

ๆThe limited examination includes general appearance, brief pulmonary, cardiac, abdominal and neurological examination.

${ }^{* *}$ These assessments are collected via cord blood.

††Malaria diagnostics include malaria rapid diagnostic test, malaria microscopy, malaria filter paper for PCR and histology at delivery.

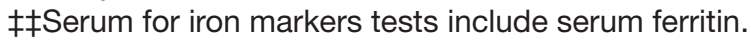

Pre-screening and screening, visit 0 (day -7 to 0 )

Women attending their first antenatal visit at ANCs are given general information (aims and procedures) about the study by a trained research nurse and invited to consent to pre-screening procedures. As the ANC nurse cares for attendees, the research nurse pre-screens potential participants by noting apparent gestation age based on clinical examination and last menstrual period and then tests for anaemia with capillary $\mathrm{Hb}$ (HemoCue 301+, Angelholm Sweden) and Plasmodium infection by rapid diagnostic test (SD Bioline Malaria AG P.F/PAN, Standard Diagnostics). Potentially eligible participants receive further information about the study. Willing participants are referred to the research site for additional evaluation, full consenting procedure and recruitment.

\section{Enrolment, visit 1 (day 0)}

As presented in table 1, at the health facility, potentially eligible participants undergo ultrasound scanning (within 2 days from the initial pre-screening visit) to confirm singleton pregnancy and determine gestation age (ie, crown-rump length for less than or equal to 16 weeks, femur length and head and abdominal circumference for more than 16 weeks). Written informed consent is then obtained for participation in the trial. For consenting participants, a physical examination is conducted, and baseline data are collected, comprising demographic information and medical and obstetric history. Following the collection of baseline clinical data, samples of both capillary and venous blood are collected and then participants are randomised. For women randomised to the FCM arm of the trial, an intravenous cannula is inserted under aseptic precautions, and a trained nurse administers the study drug according to standard procedures and under the supervision of the study clinician. Women randomised to the standard-of-care arm of the trial are provided with a full course of iron tablets, together with information delivered according to a standardised script reflecting ANC practice.

\section{Subsequent study visits}

Visit 2 (28 ( \pm 2$)$ days after enrolment into the study)

Participants receive a physical examination and an ultrasound scan before being asked for a venous blood sample (table 1). A capillary sample is collected for assessment of Plasmodium parasitaemia. The participants are asked to continue their regular ongoing ANC visits through their local health centre.

Visit 3; visits 5-6 (34 weeks' gestation \pm 2 days; $38-40$ weeks' gestation \pm 2 days)

Participants are visited in their home at 34 weeks' gestation, and every 2 weeks from 38 weeks' gestation until delivery. A research nurse measures capillary Hb. Where 
the participant belongs to the standard-of-care trial-arm, the research nurse attempts to assess adherence. Notably, since the opening of the trial, fieldwork has been affected by the COVID-19 pandemic. Because of the risks of study workers introducing COVID-19 into remote villages, home visits were removed from the trial protocol after April 2020.

Visit 4 (36 weeks' gestation/pre-delivery \pm 2 days)

Procedures are similar to those for visit 2 (28-days posttreatment). This visit includes the assessment of venous haemoglobin for the primary outcome.

Visit 7 (delivery +1 day)

The study provides 24-hour cover of the study research sites' delivery suites. All participants are asked to return to the research site for delivery (unless a high-risk pregnancy requires delivery at the tertiary referral hospital). Standardised delayed cord clamping procedures are instituted. Participants delivering at home or at other health facilities are encouraged to attend the research site for assessment within 24 hours. Venous blood samples are collected during labour. Apgar scores are recorded immediately after delivery, and the newborn undergoes a full physical examination, including measurement of birth weight, length, head circumference and assessment for congenital malformations. The type of birth and occurrence of perinatal complications (including haemorrhage or need for blood transfusion) are recorded. Placental blood is drawn, and a sample of placental tissue is collected and stored in buffered formalin for subsequent histological evaluation.

\section{Visit 8 (28 days postpartum \pm 2 days)}

Participants return to the research site together with their infants for a detailed medical examination of both mother and infant and collection of blood samples.

\section{Unscheduled visit}

Participants are asked to attend the research site when symptomatically unwell. They are managed according to national standard treatment guidelines by a trained healthcare provider. Blood samples for malaria Rapid Diagnostic Test (RDT) (and microscopy if RDT positive), and blood culture are taken if clinically indicated. The clinical diagnosis of the unscheduled visit is recorded in the participant's record. Where a participant attends another health facility or ANC, the research team extracts the missed unscheduled visit notes from the participants' health book commonly known as a health 'passport' in Malawi. Usually, clinical investigations and medications are indicated in the health book and can be extracted by the research team during the next scheduled visit.

\section{Laboratory procedures}

Venous blood is measured for haemoglobin concentration using an automated analyser (Sysmex, XP 300 series, Sysmex Corporation, Kobe, Japan), for which daily two level controls are run and recorded. Serum is separated by centrifugation and stored at $-80^{\circ} \mathrm{C}$. Samples will be batched and assayed for ferritin, $\mathrm{C}$ reactive protein and phosphate in Meander Medical Centre laboratory, accreditation number M040, EN ISO 15189:2012 (Amersfoort, The Netherlands).

\section{Data monitoring committee (DMC)}

An independent DMC has been set up to review on a regular basis, safety and efficacy data of the ongoing trial. The DMC is comprised of international experts in clinical trials, obstetrics, epidemiology and statistics. In the advent of clear evidence of effectiveness or harm, the DMC will recommend to the sponsor and investigators whether to continue, modify or terminate the trial on ethical grounds.

\section{Outcomes}

The primary outcome is the prevalence of maternal anaemia before delivery, defined as a venous haemoglobin concentration less than $110.0 \mathrm{~g} / \mathrm{L}$ at 36 weeks' gestation. This outcome evaluates the performance of the study intervention in helping women reach labour with optimal tissue oxygenation and resilience.

Secondary outcomes assessing effectiveness in the mother are anaemia at 4 weeks' post-randomisation, delivery, and 1 month postpartum, moderate/ severe anaemia and haemoglobin concentration both at 4 weeks post-randomisation, 36 weeks' gestation, delivery and 1 month postpartum, iron deficiency (ferritin $<15$ $\mathrm{mg} / \mathrm{L}$ adjusted for $\mathrm{C}$ reactive protein), iron-deficiency anaemia and serum ferritin concentration all at 4 weeks post-randomisation, 36 weeks' gestation, and 1 month postpartum.

Effectiveness in the neonate is assessed using the outcome of mean birth weight (measured in grams within 24 hours of birth) and the secondary outcomes low birth weight (birth weight less than $2500 \mathrm{~g}$ ), gestation duration (weeks), birth length, small for gestation age, fetal loss (pregnancy loss before 28 completed weeks' gestation), stillbirth (birth of a child showing no signs of life after 28 weeks' gestation), preterm births (neonate born before 37 completed weeks of gestation), haemoglobin concentration at 1 month postpartum and infant growth (weight for age, length for age, weight for length) at 1 month postpartum.

Outcomes assessing safety in the mother are (serious) AEs related to administration of FCM (events occurring during the enrolment visit), (serious) AEs (within 14 days of randomisation, antenatal and postpartum reported separately), all-cause sick visits to the clinic (antenatal and postpartum reported separately), clinical malariaspecific visits to the clinic (antenatal and postpartum reported separately), hypophosphatemia (biochemical) (at 4 weeks' post-randomisation and 36 weeks' gestation), inflammation (elevated C reactive protein) (at 4weeks' post-randomisation and 36 weeks' gestation) and severe medical events including haemorrhage, receipt of blood 
transfusion, Intensive Care Unit (ICU) admission or mortality.

Clinical infections will be reported during unplanned visits. Clinical malaria will be defined clinically, in women who present with fever and a positive malaria test. Diarrhoea will be defined in women with more than three loose stools per day. Other clinical diagnoses will be made according to local health manuals.

Safety in the infant is assessed using (serious) AEs, allcause visits to the clinic, infection-related visits to the clinic, diarrhoea-related visits to the clinic and clinical malaria-specific visits to the clinic.

In addition to the primary and secondary clinical outcomes listed above, which will support the reporting of the confirmatory RCT, data for many exploratory outcomes are being collected between enrolment and 12 months postpartum. These include assessing maternal cognition, mother-infant bonding, maternal depression and fatigue, infant neurodevelopment, infant diet and health economic data, including direct and indirect costs of healthcare. Also, women have given their consent for the collection of samples for future translational studies, including evaluation of the vaginal and faecal microbiome, maternal immune profiles and effects of interventions directed towards Plasmodium falciparum biology. Details of primary, secondary and exploratory outcomes included in the trial registration are shown in online supplemental table 1.

\section{Detection and reporting of AEs and serious AEs (SAES)}

Non-serious AEs and SAEs are collected from the time consent is given until the participant completes the study (the final visit or withdrawal). These are detected either through spontaneous reports by the participant, unplanned visits to the research site or any of the participating health centres, observation by the study staff, and through standard questioning at each visit. All SAEs are reported to the sponsor and the appropriate ethics committee, whether or not considered causally related to the study drugs.

\section{Sample size}

The sample size calculation is based on the primary maternal outcome of the proportion of women with pre-delivery anaemia (defined as venous $\mathrm{Hb}<110.0 \mathrm{~g} / \mathrm{L}$ at 36 weeks' gestation). A systematic review and metaanalysis identified a $50 \%$ reduction in anaemia prevalence in women receiving oral iron. ${ }^{2}$ In a cohort of pregnant women in the Gambia, we observed that $60 \%$ of pregnant women with $\mathrm{Hb}<100.0 \mathrm{~g} / \mathrm{L}$ at 20 weeks' gestation who then receive iron interventions continue to have $\mathrm{Hb}<100.0 \mathrm{~g} / \mathrm{L}$ by 30 weeks. ${ }^{13}$ The pivotal FERASAP (FERric carboxymaltose - Assessment of SAfety and efficacy in Pregnancy) trial (which compared FCM to oral iron in women with iron-deficiency anaemia in high-income settings) demonstrated a $14 \%$ reduction in absolute anaemia prevalence compared with oral iron. ${ }^{26}$ We hypothesise that routine iron supplementation will reduce anaemia prevalence from $100 \%$ to $60 \%$ (as seen in the Gambia, ${ }^{13}$ and similar to data from Haider and colleagues $^{2}$ ). We hypothesise that FCM will result in a $10 \%$ absolute improvement in anaemia cure (to a prevalence of $50 \%$ ). A total sample size of 862 pregnant women or 431 per group will provide $80 \%$ power to detect this difference, allowing for a $10 \%$ loss to follow-up and assuming a two-sided alpha of 5\%. The sample size also has at least $80 \%$ power to detect an absolute difference between standard-of-care oral iron and FCM of $100 \mathrm{~g}$ in the neonatal outcome of birth weight (assuming an SD of $450 \mathrm{~g}$ and a two-sided alpha of 5\%), similar to the effect size seen in a trial of Kenyan women receiving oral iron when compared with placebo. ${ }^{7}$

\section{STATISTICAL ANALYSIS PLAN}

A detailed statistical analysis plan will be finalised before unblinding of the database. Analyses will be performed where participants are classified according to their randomised intervention arm (ie, intention to treat principle). An available case analysis will be performed for repeated time point outcomes (eg, anaemia) and a complete-case analysis for single time point outcomes (eg, birth weight). Anaemia will be analysed using a log-binomial regression model, including study participants as a random intercept to account for the multiple time points. The model will include the standard-of-care (oral iron) group as the reference group. The primary maternal hypothesis will be evaluated by obtaining the estimate of the prevalence ratio of intravenous iron versus standard-of-care (oral iron), 95\% CI extracted at 36 weeks' gestation, and $\mathrm{p}$ value. Birth weight will be analysed by fitting a linear regression model. The primary neonatal hypothesis will be evaluated by estimating the absolute difference in mean birth weight between intravenous iron and standard-of-care (oral iron) along with a corresponding $95 \% \mathrm{CI}$ and $\mathrm{p}$ value. Secondary repeated time point binary outcomes will be analysed similarly to anaemia, and secondary single time point continuous outcomes will be analysed similarly to birth weight. Secondary, single time point binary outcomes (eg, suboptimal pregnancy outcomes) will be analysed using a logbinomial regression model and secondary, multiple time point continuous outcomes (eg, maternal haemoglobin concentration) will be analysed using a likelihood-based longitudinal data analysis model. ${ }^{27}$ Appropriate transformations may be applied to the variables before fitting the model if considered skewed (eg, ferritin). Additional analyses using multiple imputation will be performed to handle missing data. Results will be compared with the main analysis to investigate the findings' robustness to the missing data assumptions. Safety, including AEs, infections and clinic visits, will be presented for the mothers and neonates, respectively. The proportion of study participants with at least one safety outcome will be compared between groups using a log-binomial regression model. A Poisson model with robust standard errors will be fitted if 
there is a non-convergence of the log-binomial model for efficacy or safety outcomes. Exploratory subgroup analyses (eg, by parity, site, iron deficiency) will be performed for maternal and neonatal outcomes, irrespective of their findings. The analyses models for all study outcomes will adjust for the randomisation stratification variable of the site as a main effect.

\section{Data management}

Data collected from the subjects are recorded in digital case report forms using tablets. Relevant hard copy patient hospital files are scanned for reference and stored digitally, securely. An independent Data and Safety Monitoring Board has been established to regularly review the trial's progress and blinded and unblinded results. The Research Support Centre at the College of Medicine performs independent monitoring of the study on behalf of the sponsor. No interim analysis is planned.

\section{ETHICS AND DISSEMINATION}

The College of Medicine Research Ethics Committee (COMREC), Blantyre, Malawi, and the Walter and Eliza Hall Institute of Medical Research Human Research Ethics Committee, Melbourne, Australia, approved this study. In addition, an evaluation was done by the Regional Ethics Committee West of Norway. They advised that the study was not subject to the Norwegian Health Research Act and that ethical review from this committee was not required. The trial is approved by the Malawian Pharmacy and Medicines Regulatory Authority (PMRA/CTRC/III/25052018100). Important protocol modifications such as changes to eligibility criteria or outcomes are reported to the ethics committees, regulatory authorities in Malawi, investigators, trial participants, trial registries. Witnessed informed written consent in English or Chichewa language is obtained from each participant before conducting any study-related procedure. The study is conducted under The International Conference on Harmonisation of Technical Requirements for Registration of Pharmaceuticals for Human Use guidelines for 'good clinical practice' and the Declaration of Helsinki. The results will be presented to and shared with the local community that hosted and enabled the research, and also to the international fora. We will publish in peer-reviewed scientific journals and report to relevant policymaking bodies such as the Malawi Ministry of Health.

\section{DISCUSSION}

Maternal anaemia prevalence is highest in sub-Saharan Africa and South Asia, and current approaches to control it are failing. This trial will provide high quality, African-based evidence for clinicians, policymakers and donors on a role for modern intravenous iron for antenatal anaemia in low-income settings. This is the largest RCT programme assessing new intravenous iron formulations in pregnancy and is of major international significance in developing new global guidelines for anaemia in pregnancy. The results of this study will be disseminated to local and national medical authorities, policymakers, and be disseminated to the global research community, technical agencies and international government bodies via peer-reviewed journals and at international scientific fora.

\section{Author affiliations}

${ }^{1}$ Department of Public Health, School of Public Health and Family Medicine, University of Malawi, College of Medicine, Blantyre, Malawi

${ }^{2}$ Department of Nutrition and Infectious Diseases, Training and Research Unit of Excellence (TRUE), Blantyre, Malawi

${ }^{3}$ Centre for Epidemiology and Biostatistics, University of Melbourne School of Population and Global Health, Melbourne, Victoria, Australia

${ }^{4}$ Department of Infectious Diseases, The University of Melbourne, Melbourne, Victoria, Australia

${ }^{5}$ Population Health and Immunity Division, Walter and Eliza Hall Institute of Medical Research, Melbourne, Victoria, Australia

${ }^{6}$ Department of Global Public Health and Primary Care, University of Bergen, Bergen, Norway

${ }^{7}$ Department of Obstetrics and Gynaecology, University of Malawi, College of Medicine, Blantyre, Malawi

${ }^{8}$ Department of Medicine, Peter Doherty Institute for Infection and Immunity, University of Melbourne, Melbourne, Victoria, Australia

${ }^{9}$ Department of Obstetrics and Gynaecology, Zomba Central Hospital, Zomba, Malawi

${ }^{10}$ Malaria Alert Centre, University of Malawi, College of Medicine, Blantyre, Malawi ${ }^{11}$ Department of Health Promotion, Education, and Behavior, University of South Carolina, Arnold School of Public Health, Columbia, South Carolina, USA

${ }^{12}$ Diagnostic Haematology and Clinical Haematology, The Peter MacCallum Cancer Centre, The Royal Melbourne Hospital, Melbourne, Victoria, Australia

Twitter Martin N Mwangi @martmwangi, Glory Mzembe @MzembeGlory, Ernest Moya @ErnestMoya6, Rebecca Harding @beckyhardingnz, Julie Simpson @ JulieASimpson50, Ricardo Ataíde @ricardoataide, Kamija S Phiri @kamija_phiri and Sant-Rayn Pasricha @srpasricha

Acknowledgements We acknowledge all the study participants for their willingness to participate in this study. We appreciate the dedication of the research staff in Blantyre, Zomba and Melbourne. We are grateful for the support we continue to receive from the District Health Offices in Blantyre and Zomba districts of Malawi. We acknowledge the collaboration with the Zomba Central Hospital, especially the office of the Hospital Director. We thank Dr Alinune Kabaghe and Dr Josephine Banda, who independently packed the trial drugs and delivered them to research sites. We also acknowledge the Dr Kamiza Histopathology Laboratory for analysing the placental samples.

Contributors KSP and S-RP were involved in conception and trial design. MNM wrote the first draft of the paper. MNM, GM, EM, SB, RH and RA were involved in drafting of the article. SB and RH provided statistical expertise. MNM, GM, EM and $\mathrm{KB}$ were involved in study implementation and data acquisition. BR, JS, WS, SR, $\mathrm{JC}$ and $\mathrm{LL}$ were involved in critical revision of the article for important intellectual content. All the authors were involved in final approval of the article. Preparing study design, collection, management, analysis and interpretation of data; writing of the report; and the decision to submit the report for publication is the responsibility of the study sponsor. The study funder, the Bill and Melinda Gates Foundation, had no role in the decision to publish.

Funding This study is funded by the Bill \& Melinda Gates Foundation (OPP1169939).

\section{Competing interests None declared.}

Patient consent for publication Not applicable.

Provenance and peer review Not commissioned; externally peer reviewed.

Supplemental material This content has been supplied by the author(s). It has not been vetted by BMJ Publishing Group Limited (BMJ) and may not have been 
peer-reviewed. Any opinions or recommendations discussed are solely those of the author(s) and are not endorsed by BMJ. BMJ disclaims all liability and responsibility arising from any reliance placed on the content. Where the content includes any translated material, BMJ does not warrant the accuracy and reliability of the translations (including but not limited to local regulations, clinical guidelines, terminology, drug names and drug dosages), and is not responsible for any error and/or omissions arising from translation and adaptation or otherwise.

Open access This is an open access article distributed in accordance with the Creative Commons Attribution 4.0 Unported (CC BY 4.0) license, which permits others to copy, redistribute, remix, transform and build upon this work for any purpose, provided the original work is properly cited, a link to the licence is given, and indication of whether changes were made. See: https://creativecommons.org/ licenses/by/4.0/.

\section{ORCID iDs}

Martin N Mwangi http://orcid.org/0000-0002-8358-4448

Sabine Braat http://orcid.org/0000-0003-1997-3999

William Stones http://orcid.org/0000-0003-0699-2381

Jobiba Chinkhumba http://orcid.org/0000-0002-1921-619X

\section{REFERENCES}

1 World Health Organization. The global prevalence of anaemia in 2011. Geneva: World Health Organization, 2015.

2 Haider BA, Olofin I, Wang M, et al. Anaemia, prenatal iron use, and risk of adverse pregnancy outcomes: systematic review and metaanalysis. BMJ 2013;346:f3443.

3 Premru-Srsen T, Verdenik I, Ponikvar BM, et al. Infant mortality and causes of death by birth weight for gestational age in non-malformed singleton infants: a 2002-2012 population-based study. J Perinat Med 2018;46:547-53.

4 World Health Organization, United Nations Children's Fund, United Nations University. Iron deficiency anaemia: assessment, prevention, and control. A guide for programme managers. Geneva: World Health Organization, 2001.

5 Daru J, Zamora J, Fernández-Félix BM, et al. Risk of maternal mortality in women with severe anaemia during pregnancy and post partum: a multilevel analysis. Lancet Glob Health 2018;6:e548-54.

6 Peña-Rosas JP, De-Regil LM, Garcia-Casal MN, et al. Daily oral iron supplementation during pregnancy. Cochrane Database Syst Rev 2015;170:CD004736.

7 Mwangi MN, Roth JM, Smit MR, et al. Effect of daily antenatal iron supplementation on Plasmodium infection in Kenyan women: a randomized clinical trial. JAMA 2015;314:1009-20.

8 World Health Organization. Iron and folate supplementation. integrated management of pregnancy and childbirth (IMPAC). Geneva: World Health Organization, 2006.

9 World Health Organization. Essential nutrition actions: improving maternal, newborn, infant and young child health and nutrition. Geneva: World Health Organization, 2013.

10 Low MSY, Speedy J, Styles CE, et al. Daily iron supplementation for improving anaemia, iron status and health in menstruating women. Cochrane Database Syst Rev 2016;4:CD009747.
11 Stoffel NU, Cercamondi CI, Brittenham G, et al. Iron absorption from oral iron supplements given on consecutive versus alternate days and as single morning doses versus twice-daily split dosing in iron-depleted women: two open-label, randomised controlled trials. Lancet Haematol 2017;4:e524-33.

12 National Statistical Office (NSO) [Malawi], ICF International. Malawi demographic and health survey 2015-16: key indicators report. Zomba, Malawi, and Rockville, Maryland, USA: NSO and ICF International, 2016.

13 Bah A, Pasricha S-R, Jallow MW, et al. Serum hepcidin concentrations decline during pregnancy and may identify iron deficiency: analysis of a longitudinal pregnancy cohort in the Gambia. J Nutr 2017;147:1131-7.

14 Mkandawire P. Gestational age at first antenatal care visit in Malawi. Matern Child Health J 2015;19:2366-74.

15 Friedrisch JR, Cançado RD. Intravenous ferric carboxymaltose for the treatment of iron deficiency anemia. Rev Bras Hematol Hemoter 2015;37:400-5

16 Pasricha S-R, Tye-Din J, Muckenthaler MU, et al. Iron deficiency. The Lancet 2021;397:233-48.

17 Shand AW, Bell J, Henry A, et al. Rapid increase in intravenous iron therapy for women of reproductive age in Australia. Med $J$ Aust 2020;213:85-6.

18 Qassim A, Grivell RM, Henry A, et al. Intravenous or oral iron for treating iron deficiency anaemia during pregnancy: systematic review and meta-analysis. Med J Aust 2019;211:367-73.

19 Achebe MM, Gafter-Gvili A. How I treat anemia in pregnancy: iron, cobalamin, and folate. Blood 2017;129:940-9.

20 Neogi SB, Devasenapathy N, Singh R, et al. Safety and effectiveness of intravenous iron sucrose versus standard oral iron therapy in pregnant women with moderate-to-severe anaemia in India: a multicentre, open-label, phase 3, randomised, controlled trial. Lancet Glob Health 2019;7:e1706-16.

21 Ortiz R, Toblli JE, Romero JD, et al. Efficacy and safety of oral iron(III) polymaltose complex versus ferrous sulfate in pregnant women with iron-deficiency anemia: a multicenter, randomized, controlled study. $J$ Matern Fetal Neonatal Med 2011;24:1347-52.

22 Vanobberghen F, Lweno O, Kuemmerle A, et al. Efficacy and safety of intravenous ferric carboxymaltose compared with oral iron for the treatment of iron deficiency anaemia in women after childbirth in Tanzania: a parallel-group, open-label, randomised controlled phase 3 trial. Lancet Glob Health 2021:9:e189-98.

23 Bank W. Prevalence of low birthweight babies (\% of births) - Malawi Washington DC, USA2021 [21 August 2021]. Available: https://data. worldbank.org/indicator/SH.STA.BRTW.ZS?locations=MW

24 Boudová S, Divala T, Mawindo P, et al. The prevalence of malaria at first antenatal visit in Blantyre, Malawi declined following a universal bed net campaign. Malar J 2015;14:422.

25 Young MF, Oaks BM, Tandon S, et al. Maternal hemoglobin concentrations across pregnancy and maternal and child health: a systematic review and meta-analysis. Ann N Y Acad Sci 2019;1450:47-68.

26 Breymann C, Milman N, Mezzacasa A, et al. Ferric carboxymaltose vs. oral iron in the treatment of pregnant women with iron deficiency anemia: an international, open-label, randomized controlled trial (FER-ASAP). J Perinat Med 2017;45:443-53.

27 Liang K-Y, Zeger SL. Longitudinal data analysis of continuous and discrete responses for pre-post designs. Sankhyā: The Indian Journal of Statistics 2000; Series B:134-48. 